\title{
SUL VENTO PREVALENTE A PAVIA
}

\author{
G. Aliverti - E. Avico
}

Ripctutamente $\dot{c}$ capitato a una li noi. negli anni scorsi, di ricevere richieste di dati da persone interessate per $\mathrm{i}$ più svariati fini a conoscere le caratteristiche principali del vento di una determinata zona d'Italia. Per quanto riguarda Pavia si è rilevato così che mancava una elaborazione dei dati del vento che tenesse conto dei due elementi, direzione ed intensita; per quelle informazioni si poteva ricorrere soltanto o alla climatoloția del Roster o al lavoro del prof. Eredia, per il decennio 1891-1900, lavori che considerano la direzione e non l'intensitit. Ora la osservazione quotidiana delle carte del vento, otlenute all'Osservatorio dopo il 1942, cioè dopo l'installazione di un ottimo anemografo meccanico Siap, ha fatto nascere il sospetto in una di noi che $\mathrm{i}$ dati riassuntivi a disposizione e relativi a Pavia rispecchiassero una distribuzione del vento nelle varie direzioni notevolnente diversa da quella che ci si poteva aspettare dalla elaborazione completa dei nuovi dati. Appariva dalla semplice ispezione a occhio delle carte che una direzione, il $\mathrm{SW}$, era di gran lunga la preferita.

Decidenmo perciò la elalorazione di tutti i dati registrati durante le 24. ore c la hasammo sulle seguenti considerazioni: siccome una delle caratteristiche più interessanti praticamente è la direzione prevalente e siccome il vento ha la tendenzal a cambiare bruscamente direzione ma poi persiste nella direzione assunta abbastanza a lungo, se si suddivide la reģistrazione di ogni giornata per direzioni e si contano in corrispondenza i chilometri di vento filato e il tempo trascorso, si può ottenere facilmente uno specchio della inportanza dei vari venti. La suddivisione diventa un po' faticosa soltanto in occisione di temporali, durante i quali si hanno variazioni frequenti e ampie; ma altrimenti capita in generale che un vento tiene il campo per ore ed ore consecutive. A Pavia ciò si verifica in modo particolarmente vistoso per il $\mathrm{SW}$ e in nisura notevole anche per il NF. Ci siamo accontentate di considerare otto direzioni, perché effettivamente, dato il carattere del vento, la suddivisione in sedici parti ci parve eccessiva e un numero intermedio di direzioni non era possibile 
considerarlo date le carte diagrammate a disposizione. Gli anni considerati sono sette e precisamente dal 1943 al 1949; durante quasi tutto quel periodo, per cause contingenti relative alla guerra, l'Osservatorio eblue scarsità di carte diagrammate e di conseguenza ogni carta fu in generale usata almeno due volte e magari anche tre con inchiostri di colore diverso; questo fatto ha reso talvolta un po' penosa la elaborazione e purtroppo ci ha fatto perdere qualche centinaio di ore di registrazione. Dell'inverno $1946-47$ estremamente rigido si perdettero alcune giornate di registrazione per cattivo funzionamento dell'orologio; in complesso le ore utilizzate sono state quindi 60474 invece delle 61368 costituenti i sette anni considerati.

Siccome gli Uffici Centrali Europei di Meteorologia usano ancora raccogliere nel "registro delle stazioni " i dati del vento relativi alle tre ore soltanto delle osservazioni (cioè delle ore $8,14,19$ ), e si vedono anche in pubblicazioni recenti elaborazioni basate sull'uso di quei dati relativi a tre sole ore fisse della giornata, abbiamo eseguito anche noi una elaborazione di quel tipo per poterne confrontare i risultati con quelli delle 24 ore.

Nella tabella I sono raccolti per l'elaborazione estesa alle ventiquattro ore: i chilometri totali per ciascuno degli otto venti, la corrispondente durata in ore, la velocita media in $\mathrm{km} / \mathrm{h}$ dedotta dividendo il numero dei chilometri per il numero delle ore, e infine il numero di volte in cui ciascun vento ha soffiato; nella penultima orizzontale sono indicate le ore totali di calma e il numero delle volte in cui la calma si è verificata; nell'ultima orizzontale ci sono le somme

\section{TABELLA I}

\begin{tabular}{|c|c|c|c|c|c|c|}
\hline \multicolumn{5}{|c|}{ Dalle registrazioni delle 24 ore (1943.19 Pavia) } & \multicolumn{2}{|c|}{ Dalle tre ore $(8,14,19)$} \\
\hline vento & $\begin{array}{c}\text { percorso } \\
\text { km }\end{array}$ & $\begin{array}{l}\text { durata tot. } \\
\text { ore min }\end{array}$ & $\left|\begin{array}{c}\text { vel. media } \\
\mathrm{km} / \mathrm{h}\end{array}\right|$ & volte & $\begin{array}{c}\text { percorso } \\
\text { km }\end{array}$ & $\begin{array}{l}\text { volte } \\
(=\text { ore })\end{array}$ \\
\hline $\mathbf{N}$ & 28789 & 466252 & 6.2 & $2 \mathrm{I} 73$ & 6231 & 886 \\
\hline NE & 59232 & 1199923 & 49 & +953 & 3843 & 837 \\
\hline $\mathbf{E}$ & $3+1333$ & 662238 & 5,2 & 3973 & 8394 & 153.1 \\
\hline SE & 22902 & 418859 & 5,6 & 3288 & 1361 & 235 \\
\hline $\mathrm{S}$ & 6492 & 197645 & 3.3 & 1378 & 2360 & 636 \\
\hline SW & 114811 & 16181is U(; & 6.4 & 54113 & $9621:$ & 1417 \\
\hline W & 32487 & 654135 & 5,0 & 3872 & 9.10 & $15 \div 1$ \\
\hline NW & 24125 & $105+28$ & 5,9 & 2598 & 1345 & 229 \\
\hline calma & 一 & $236+06$ & - & 2847 & - & 79 \\
\hline totale & 323171 & 6017352 & $5,3 \quad(5,6) \mid$ & 30185 & 42570 & 747.4 \\
\hline
\end{tabular}


dei chilometri e della durata e la velocità media corrispondente; in parentesi la velocità media calcolata escludendo le ore di calma. Nelle ultime due colonne della tabella I sono poi raccolti per il confronto i risultati della elahorazione relativa alle tre ore delle osservazioni e precisamente i chilometri totali e il corrispondente numero di volte (= ore) in cui ciascun vento è stato osservato.

A proposito della claborazione cstesa alle 24 ore ̀̀ da notare che

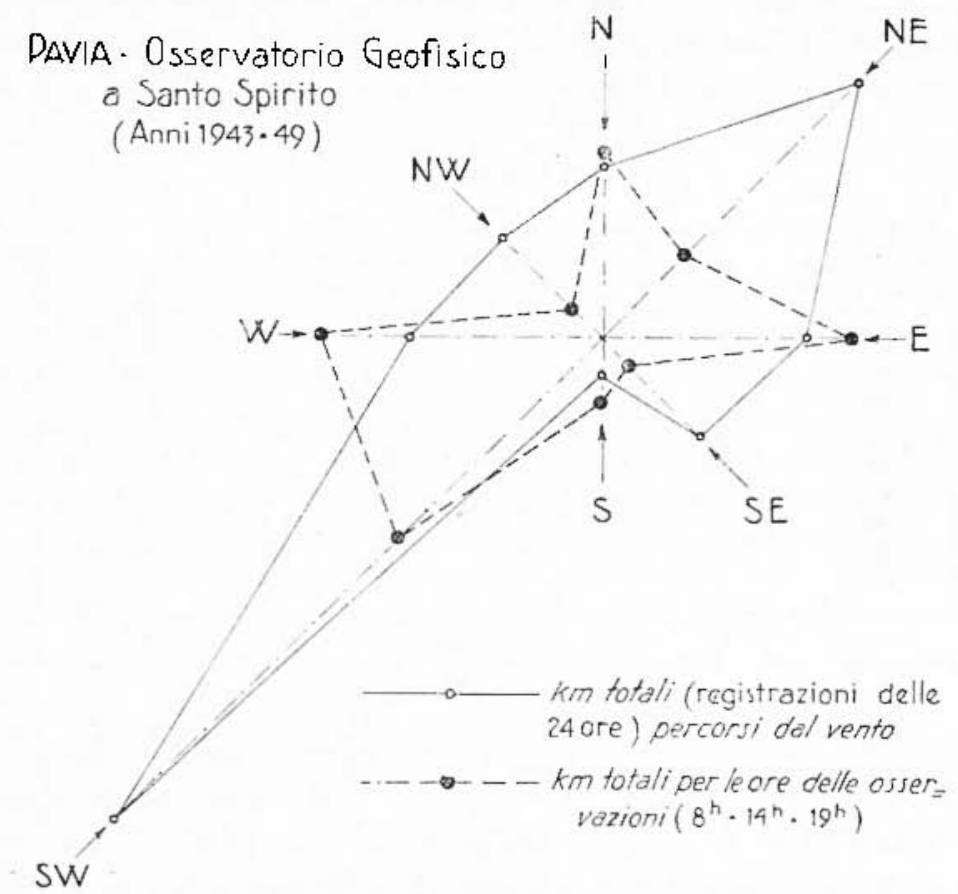

Fig. 1

i risultati di un singolo anno si scostano poco da quelli ottenuti dai 7 anni insieme; così per es. il SW risulta in og̣ni anno il vento più importante, pex il numero dei $\mathrm{km}$ e il numero delle ore, mentre il $\mathrm{S}$ risulta sempre il meno importante. Ciò non accade per l'claborazione dei dati delle tre ore $e$ su sette anni elaborati soltanto tre danno la prevalenza del SW per i $\mathrm{km}$ percorsi, e il $\mathrm{S}$ soltanto due volte risulta il vento meno importante. Si può così concludere che tenendo conto di tutte le 24 ore, il cosidetto periodo normale (valevole per il calcolo della distribuzione normale) è già verosimilmente raggiunto con l'intervallo di sette anni, mentre ciò non avviene sicuramente per la ela- 
borazione delle tre ore che comprende soltanto l'ottava parte del tempo tenuto in conto nella prima elaborazione, e già per questa ragione essa richiederebbe un numero di anni almeno otto volte più grande; inoltre le tre ore scelte, significative per altri elementi meteorologici, non lo sono di sicuro per il vento; l'elaborazione in parola darebbe risultati migliori certamente se le tre ore fossero scelte a caso entro le 24 ore. Il confronto fra i numeri della seconda e della sesta colonna della tabella I mette subito in evidenza la differenza sostanziale fra i risultati delle due elaborazioni; gli stessi numeri sono rappresentati graficamente nella fig. I che rende il confronto più efficace; le unità

Tabella II

\begin{tabular}{|l|c|c|c|}
\hline \multirow{2}{*}{ vento } & \multicolumn{3}{|c|}{ Importanzal riguardo a: } \\
\cline { 2 - 4 } & percorso & durati & volte \\
\hline N & $9 \%$ & $7 \%$ & $7 \%$ \\
NE & 18 & 20 & 16 \\
SE & 11 & 11 & 13 \\
S & 7 & 7 & 11 \\
SW & 2 & 3 & 5 \\
W & 36 & 11 & 5 \\
NW & 10 & 71 & 13 \\
\hline calma & 7 & 7 & 8 \\
\hline
\end{tabular}

usate per le due rappresentazioni sono naturalmente differenti fra loro. I venti di $\mathbf{W}, \mathbf{E}, \mathbf{N}$, di moderata importanza eflettiva, nella elaborazione delle tre ore assumono importanza pari o quasi al $\mathrm{SW}$ che è il vento nettamente prevalente; perciò non abbiamo elaborato ulteriormente tali dati.

Nella tabella II sono riportate le percentuali relative alle otto direzioni rispetto ai $\mathrm{km}$ totali, alle ore totali e al numero di volte totali; i numeri di questa tabella, ricavati dalla tabella I, graduano cioè l'importanza di ciascun vento; per la durata e le volte si è tenuto in conto anche la calma.

Come si vede il SW è il vento più importante per i chilometri percorsi, per la durata e anche per il numero di volte in cui ha soffiato; il $S$ è invece il meno importante.

Le tabelle I e II si riferiscono ai risultati complessivi dei sette anni; ma è interessante anche vedere la distribuzione nelle stagioni. La tabella III riassume tali dati. 
TABELLA III

\begin{tabular}{|c|c|c|c|c|c|c|c|c|}
\hline \multirow{2}{*}{ Vento } & \multicolumn{2}{|c|}{ Inverno } & \multicolumn{2}{|c|}{ Primavera } & \multicolumn{2}{|c|}{ Estate } & \multicolumn{2}{|c|}{ Autunno } \\
\hline & $\mathrm{km}$ & durata & $\mathrm{km}$ & durata & $\mathrm{km}$ & durata & $\mathrm{km}$ & durata \\
\hline $\mathbf{N}$ & 7450 & $1014^{\mathrm{l}} 00^{\mathrm{mu}}$ & 9981 & $1173^{\mathrm{h}} 17^{\mathrm{m}}$ & 6908 & $1.452^{11}+8^{\mathrm{m}}$ & .4.50 & $1022^{\mathrm{h}}, 2^{\mathrm{m}}$ \\
\hline NE & 9980 & 172933 & 18715 & $2915: 40$ & 16398 & 4111743 & 1.4139 & 333627 \\
\hline E & 6477 & 103942 & 13235 & 222352 & 7064 & $165] 24$ & 7557 & 170740 \\
\hline SE & 2740 & 49455 & 9247 & 145335 & 6123 & 128826 & 4792 & 95203 \\
\hline $\mathrm{s}$ & 1838 & 53.50 & 2005 & 54.45 .5 & 1015 & 33531 & 1634 & 56129 \\
\hline SW & 42977 & 699330 & 333819 & 435110 & $2011+15$ & 306032 & 179)(4) & 36.5754 \\
\hline W & 8657 & 183322 & 8482 & 1.12120 & 8621 & 158639 & 7124 & 170014 \\
\hline NW & 6599 & 7.1700 & 5935 & 81110 & 6716 & 132154 & 487.5 & 11742.1 \\
\hline ralma & - & 49913 & - & 31726 & - & 54215 & - & $1005 \quad 12$ \\
\hline
\end{tabular}

Anche in questa tabella sono sottolineati $i$ valori massimi e $\mathrm{i}$ minimi; si vede bene da essa che per i chilometri percorsi il SW conserva la prevalenza ( $\left.{ }^{1}\right)$ in tutte le quattro stagioni, quantunque il distacco dagli altri venti si attutisca man mano passando dall'inverno alla primavera, dalla primavera all'estate e dall'estate all'autunno; il NE nell'estate ha la prevalenza per il numero di ore, ma sta al $2^{\circ}$ posto per i chilometri. Pure il S per i chilometri percorsi rimane sem-

\section{Tabella IV}

\begin{tabular}{|c|c|c|c|c|}
\hline Fpoca & kn totali & durata totale & ore di calma & $\begin{array}{l}\text { relocità } \\
\text { medìa }\end{array}$ \\
\hline $\begin{array}{l}\text { Inverno } \\
\text { Primavera } \\
\text { Estate } \\
\text { A utunno }\end{array}$ & $\begin{array}{r}86718 \\
100519 \\
73462 \\
62471\end{array}$ & $\begin{array}{l}1+386^{15} 52^{\mathrm{m}} \\
1489459 \\
1471457 \\
1411258\end{array}$ & $\begin{array}{rl}499 & 13 \mathrm{~m} \\
317 & 26 \\
542 & 15 \\
1005 & 12\end{array}$ & $\begin{array}{l}6,0 \mathrm{~km} / \mathrm{h} \\
6,7 \\
5,0 \\
4,4\end{array}$ \\
\hline A & 323171 & $5810946^{\mathrm{m}}$ & $\overline{2364^{1}} \sqrt{106^{m}}$ & $5,6(5,3) \mathrm{km} / \mathrm{h}$ \\
\hline
\end{tabular}

pre il meno importante. Nella tabella IV sono raccolti i dati complessivi relativi alle quattro stagioni senza distinzione di direzione; da essa si vede come la prinavera è la stagione con il numero massimo di chilometri percorsi dal vento e con la maggiore velocità media; ad essa seguono per importanza di percorso e di velocità media

(1) Prevalenza evidententente determinata dalla circolazione generale dell'atmosfera. 
nell'ordine: inverno, estate, autunno. L'autunno spicca sulle altre stagioni per il maggior numero di ore di calma.

Per quanto riguarda l'elaborazione di Ercdia citata più su, è da tener presente cbe $i$ dati da lui usati erano stati raccolti nell'Osservatorio Meteorologico dell'Universiti, sulla torretta dell'edificio universitario situato al centro della cittì. Essa dà nell'anno i seguenti valori di frequenza per le otto direzioni:

$\begin{array}{ccccccccc}\text { N } & \text { NE } & \text { E } & \text { SE } & \text { S } & \text { SW } & \text { W } & \text { NW } & \text { calma } \\ 101 & 204 & 158 & 127 & 81 & 175 & 215 & 98 & 41\end{array}$

In questa elaborazione (di dieci anni delle tre osservazioni giornaliere), per la frequenza prevale dunque l'W seguito da NE, SW, E, SE, N, NW, S. Nella analoga elaborazione dei dati raccolti da noi a Santo Spirito, e riprodotti nella tabella $I$, ultime due colonne, i venti per i chilometri percorsi si ordinano cosi: $S W, W, E, N, N E$, S, SE, NW e i chilometri percorsi dall'W sono di poco inferiori a quelli del SW. L'ordine dei venti per la frequenza risulta invece il seguente: W, E, SW, N, NE, S, SE, NW; dunque anche a Santo Spirito $l^{\prime} W$ risulterebbe il vento più frequente, giusta $i$ risultati della elaborazione dell'Eredia.

Però anche per la claborazione dell'Eredia si possono ripetere le considerazioni già svolte a proposito della nostra elahorazione dei dati delle tre ore.

Concludendo si può sottolineare cbe per ottenere $\mathrm{i}$ valori normali della velocità e della frequenza del vento, l'elahorazione dei dati delle 24 ore consente l'uso di un periodo di tempo non tanto lungo; perciò anche da questo punto di vista è desiderabile di elaborare sempre tutti i dati forniti dalle registrazioni e non soltanto quelli delle tre ore, tanto più che cosi facendo è più facile conseguire l'omogeneita del materiale elaborato.

Napoli - Istituto Superiore Navale - Gennaio 1952.

\section{RIASSUNTO}

Nella presente nota si rende conto della elaborazione dei dati del vento registrati a Pavia presso l'Osservatorio dell'Istituto Nazionale di Geofisica durante sette anni (1943-49). L'elaborazione è stutu eseguita annotando per ogni giorno e per le otto direzioni i chilometri percorsi dal vento e la durata di ciuscun vento. Da questa elaborazione si è rica- 
vata poi la velocità media di ciascun vento e l'importanza relativa per i chilometri percorsi, per la durata e per il numero di volte in cui ciascun vento si ̀̀ presentato. Sono pure stati suddivisi $i$ dati per stagione e si ̀̀ così dimostrato che il vento prevalente a Pavia è sempre il SIV per quanto riguarda $i$ chilometri percorsi e quasi sempre anche per le ore di durata (fa eccezione l'estate).

Si paragona poi tale elaborazione con $i$ risultati della elaborazione dei dati osservati alle ore 8, 14, 19, per dimostrare la inefficienza di quest'ultimo metodo di elaborazione, che di tanto in tanto viene ancora adoperato.

\section{SUMMARY}

Account is given with the present paper about the elaboration of wind data recorded in Pavia, Istituto Nazionale di Geofisica Observatory, during seven years (1943-49). The elaboration has been done registering, for every day and for the eight directions, the kilometers the wind travelled and the time each wind blew. From this elaboration has been draun the mean velocity of each wind and the importance of them for the kilometers travelled, the time over which they blew, and, at last, for the number of times during which every one occurred. The data are likevise selected according to the seasons and it has been shown that the prevailing wind in Pavin is always the SI-wind as what is concerned with the kilometers travelled, and almost always for the duration (the summer is the exception).

After all these results have been compared with those of elaboration of data observed at 8, 14, 19 hours in order to show the inefficiency of this last method of elaboration. 